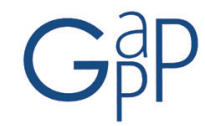

GESTIÓN Y ANÁLISIS DE POLÍTICAS PÚBLICAS, Nueva Época, nº 12 julio-diciembre 2014 ISSN: 1989-8991

DOI: http://dx.doi.org/10.24965/gapp.voi12.10212

\title{
Confianza, instituciones informales y políticas públicas, una compleja relación pendular
}

\author{
$M^{\mathrm{a}}$ Cecilia Güemes \\ Grupo de Investigación en Gobierno, Administración y Políticas Públicas GIGAPP \\ Investigadora García Pelayo, Centro de Estudios Políticos y Constitucionales (CEPC) \\ cecilia.guemes@gigapp.org \\ José Hernández-Bonivento \\ Instituto Chileno de Estudios Municipales. Universidad Autónoma de Chile \\ Investigador GIGAPP (Grupo de Investigación en Gobierno, Administración y Políticas Públicas) \\ jhernandez@ichem.cl \\ Recibido: 15 de abril 20/4 \\ Aceptado: I septiembre 2014
}

Resumen

Estudiosos en la materia han señalado una relación bidireccional entre el desempeño de políticas públicas y el contexto social en el que se desenvuelven. En esta vía, el texto busca acercarse y analizar aspectos específicos de dicha relación, la cual es descrita de manera pendular: por un lado, el enfoque dado a las políticas públicas logra afectar la confianza entre las personas de una comunidad, así como sus lazos relacionales; por el otro, la informalidad contextual donde se implementan afecta el resultado mismo de las políticas públicas. Para ello, se analizan los resultados de dos investigaciones: el efecto de las políticas públicas mercantilistas en la confianza social en Argentina y la influencia de las instituciones informales en el proceso de descentralización en Colombia. Se concluye manifestando la importancia de este acercamiento en el contexto latinoamericano, donde existen diferencias entre formalidad e informalidad, y donde la desconfianza en las personas y en las instituciones sigue siendo alta, y la necesidad de ocupar este tema en la agenda de investigación en la región.

Palabras clave

Confianza, instituciones informales, comportamiento social, políticas públicas, Latinoamérica.

\section{Trust, informal institutions and public policies, a complex pendular relationship}

\section{Abstract}

Scholars have point out the complex and bidirectional relationship between trust, informal institutions and public policies. The effective implementation of public policies depends on the level of social and institutional trust and on informal institutions, at the same time that trust and social practices are affected by public policies. This paper analyse this pendulum relationship on Latin America through the results of two researches: the effects of policies on social trust in Argentina and the influence of informal institutions in the decentralization of Colombia. It concludes by pointing out the relevance of this subject for public policy studies in Latin America, due to the great differences between formality and informality and the low rates of social trust in the region.

Keywords

Trust, Informal Institutions, Social Behaviour, Public Policies, Latin America 


\section{INTRODUCCIÓN}

El fracaso de ciertas reformas políticas en América Latina ha conducido a académicos y actores políticos a revisar como las trayectorias históricas, la cultura y lo no formal afecta e influye en cómo se desenvuelven las políticas públicas. En este contexto, temas como el capital social y las instituciones informales comienzan a ganar relevancia académica y política como claves para entender las sociedades y los efectos (o falta de efecto) de las políticas públicas (Burky \& Perry, 1998; Ostrom \& Ahn, 2003; North, 1993; Stiglitz, Sen \& Fitoussi, 2008).

Partiendo de esto, el objetivo principal del presente texto es realizar una aproximación teórica y práctica sobre la compleja relación entre las políticas públicas y el contexto social en el que se implementan. Se observaran con especial interés dos puntos que, en los últimos años, han venido desarrollándose como ejes de investigación en la región latinoamericana: la confianza y las instituciones informales. A tal fin, se recogen los resultados de dos investigaciones que dividen el presente artículo: por un lado, la primera está centrada en cómo las políticas públicas afectan la confianza social en el caso argentino; por el otro, en una segunda parte, el artículo se enfoca en la preexistencia de instituciones informales competitivas y su influencia en la implementación de la descentralización colombiana. De esta forma se busca observar la relación pendular que existe entre el contexto social y las políticas públicas.

Resulta evidente que entender esta doble relación significaría una herramienta fundamental para que el diseño, la implementación y la evaluación de políticas públicas integren elementos contextuales que permitan alcanzar, de la manera más directa posible, las metas sociales, económicas y políticas que implica el desarrollo de la región latinoamericana. Con la expectativa de colaborar en esta vía, se expondrán a continuación los aspectos teóricos en los que se afianza el artículo para luego realizar un acercamiento empírico a los casos mencionados, para concluir con una serie de reflexiones e interrogantes para nuevos acercamientos en la materia.

\section{APORTES TEÓRICOS}

El concepto de capital social ha logrado una creciente popularidad en los últimos años, pues a pesar de ser un término que busca enmarcar fenómenos difíciles de estudiar, medir y operacionalizar, es también un esfuerzo por teorizar alrededor de situaciones informales de clara relevancia en el desarrollo y desempeño de los gobiernos (Putnam, 1993 y 2001; Evans, 1996; Bowles \& Gintis, 2001; Ostrom \& Ahn, 2003).

La primera y más famosa definición de capital social, la da Putnam (1993:167), quien lo describe como rasgos de la organización social que involucran la confianza social, las reglas y las redes y que son relevantes para explicar la mayor eficacia de las sociedades y facilitar las acciones coordinadas. En torno a esta polisémica y discutida conceptualización del capital social, destaca la confianza social. Entendida alternativamente como sinónimo, elemento, producto y/ o causa del capital social, la confianza social remite a los lazos débiles o de largo alcance que se desarrollan entre grupos y personas que carecen de conocimiento íntimo entre sí y que pueden tener distinta identidad y diferentes grados de poder sociopolítico. Así, la confianza social se distingue analíticamente de la confianza singularizada, esto es: lazos fuertes y que se gestan con la familia o amigos, y de la denominada confianza institucional o vertical, que refiere a la confianza en las instituciones sociales, sean estas gubernamentales (tales como el Congreso, Poder Judicial, la policía o el Presidente) o no gubernamentales (la Iglesia, los sindicatos, o los partidos políticos) (Granovetter, 1973; Rothstein 2000; CEPAL, 2002; Levi, 1998).

Un segundo concepto por describir es el de instituciones informales, descritas por North (1993), como aqueIlas reglas que gobiernan los comportamientos por fuera de los canales oficiales. Helmke \& Levitsky (2006) las describen como reglas sociales compartidas, usualmente no escritas que son creadas y ejercen poder por fuera de los canales oficiales de sanción, que son de público conocimiento y presuponen la expectativa generalizada de que todos las seguirán. Conocidas y evidentes para los actores sociales, son sin embargo difíciles de precisar y medir justamente por su informalidad. Al igual que las reglas formales, tales instituciones descansan en un sistema de sanciones y poder que provee incentivos a los sujetos para que se comporte o no de ciertos modos (Brinkerhoff \& Goldsmith, 2002; Helmke \& Levitsky, 2006; Guarneros-Mesa, 2009).

Presentadas las definiciones, este trabajo buscará analizar la relación de la confianza e instituciones informales con las políticas públicas, entendiendo a estas últimas como un programa de acción específico de una o más autoridades de gobierno dentro de un sector de la sociedad o área de acción (Mény \& Thoenig, 1992).

En materia de confianza se sostiene que altos niveles de confianza social e institucional predicen políticas públicas más eficaces. Esto sucedería porque el capital social y la confianza hacen más probable la cooperación entre burócratas, entre elites de gobierno y entre el Estado y la sociedad civil, y como se ha sostenido, esta cooperación 
entre actores está íntimamente relacionada con el buen desempeño gubernamental y con mayor eficacia, eficiencia y legitimidad de la intervención estatal. Asimismo, se sostiene que la confianza social convierte a los ciudadanos en sofisticados de la política, que los vuelve más demandantes y controladores pero también más virtuosos y participativos, todo ello facilita la articulación de las demandas sociales y potencia el compromiso y la cooperación entre burócratas y ciudadanía (Putnam, 1993; Evans, 1996; Boix \& Postner, 2000; Rothstein \& Uslaner, 2005). ${ }^{1}$

Respecto de las instituciones informales, se afirma que las mismas pueden reforzar, subvertir y a veces reemplazar a las reglas y procedimientos formales, de modo que la formulación, implementación y los efectos de las políticas públicas estarán mediados y delineados por las instituciones informales que existan previamente (Helmke \& Levitsky, 1996). De hecho, dado que la mayoría de las reglas de comportamiento son autoimpuestas y que las reglas formales solo ocupan una proporción menor como guías de la acción cotidiana, las reglas informales y costumbres suelen ser determinantes para entender los comportamientos (Ensminger, 1997; Greif, 1997; Zenger et al, 2001; Brinkerhoff \& Goldsmith, 2002; North, 2003; Prats-Cabrera, 2008; Guarneros-Mesa, 2009).

Mirando la relación desde el otro lado, sobre el impacto de las políticas públicas en la confianza, se encuentran diferentes abordajes. Por un lado, aquellos trabajos que consideran relevante el papel de las políticas públicas en educación, formación cívica y fomento del asociacionismo, en tanto estas estimulan el desarrollo de la virtud y los lazos horizontales, crean confianza social (Almond \& Verba, 1970; Linares, 2007; Herreros \& Criado, 2001). En otra línea, buena parte de la literatura considera que las políticas de transparencia, lucha contra la corrupción, acceso a la información pública, apertura de gobierno, participación ciudadana y accountability son fundamentales para incrementar la confianza institucional y social en tanto crean predictibilidad y reducen los riesgos de confiar (Evans, 2003; Rothstein \& Stolle, 2002; Rothstein \& Uslaner, 2005; Offe, 1999). Por último, pero no menos importante, las políticas redistributivas y en pos de la igualdad de oportunidades también contribuirían a la confianza social en tanto eliminan diferencias y combaten la exclusión, cuestiones estas negativamente relacionadas con la confianza (Kumlin \& Rothstein, 2003; Rothstein, 2008; Patulny, 2005). Además de estas cuatro vías, en este trabajo consideraremos que las políticas públicas influyen indirectamente en la creación de la confianza en tanto dan forma a los escenarios en donde ésta se genera. La confianza depende a nuestro entender de cómo son las estructuras sociales (más o menos igualitarias), cuales imaginarios predominan (más o menos solidarios) y qué características tienen los espacios públicos de socialización (más o menos abiertos). El modo en que las políticas públicas influyen en tales escenarios, define las mayores o menores probabilidades que tiene la confianza (Güemes, 2011).

Sobre el margen de transformación que tienen las políticas públicas en las instituciones informales, Helmke \& Levitsky (2006:22-25) mencionan tres vías en virtud de las cuales las instituciones informales pueden ser alteradas: a través de cambios en la institucionalidad formal, por medio de la distribución del poder y de recursos entre actores sociales, o por cambios en creencias compartidas y experiencias colectivas. El presente trabajo se enfoca en la primera situación, dado el interés manifiesto por estudiar el efecto de las políticas públicas. Así se argumentará que los cambios en el diseño y efectividad de las instituciones formales (leyes y políticas) puedan afectar las instituciones informales en tanto cambien el balance entre costos y beneficios entre unas u otras. Si seguir las reglas formales se considera más efectivo y al mismo tiempo seguir las informales se vuelve desventajoso para los intereses y objetivos de los sujetos, es de esperar que las reglas formales se conviertan en el mejor curso de acción. Ello podría ocurrir de dos modos, o bien porque la efectividad de las formales se refuerzan o bien porque las informales pierden peso o incentivos.

En esencia, es observable una relación bidireccional entre las políticas públicas y la informalidad contextual en la que se desarrollan, la cual se comporta a manera de un péndulo, donde tanto las políticas afectan el contexto como el contexto afecta a las políticas y sus resultados. A manera de ilustrar esta relación, a continuación nos centraremos en el escenario latinoamericano, donde el desafío de apertura y modernización de los gobiernos se impone sin a veces reflexionar sobre el panorama sobre el cual se debe actuar previa o simultáneamente.

\section{AVANCES EMPÍRICOS}

\subsection{Pinceladas latinoamericanas}

En este apartado se describen algunos rasgos de la región destacando en términos comparados los bajos niveles de confianza social e institucional que la caracterizan y los altos niveles de clientelismo y anomia. Luego, se presentan los resultados de las investigaciones realizadas sobre la relación pendular que existe entre confianza social, instituciones y políticas públicas en dos sociedades latinoamericanas: la argentina y la colombiana.

1 Frente al optimismo por el capital social y la confianza es preciso destacar que no todo capital social es valioso o positivo socialmente ni tampoco siempre fungible, intercambiable o trasladable desde un espacio a otro Ver: Portes, 1998; Levi, 2001y Piselli, 2003 entre otros. Se agradece al árbitro anónimo por recordar tal cuestión. 
Sobre lo primero, como indican los Gráficos 1 y 2, América Latina es la región a nivel mundial con menores niveles de confianza social y confianza en las instituciones encargadas de hacer cumplir y aplicar las leyes (policía, tribunales y administración pública). La confianza en la Administración pública, como ilustra la figura 3, también es muy baja en la región no superando la media del 36\%.

\section{GRÁFICO 1}

CONFIANZA POR REGIONES, PROMEDIOS GENERALES. AÑOS 2007-2008

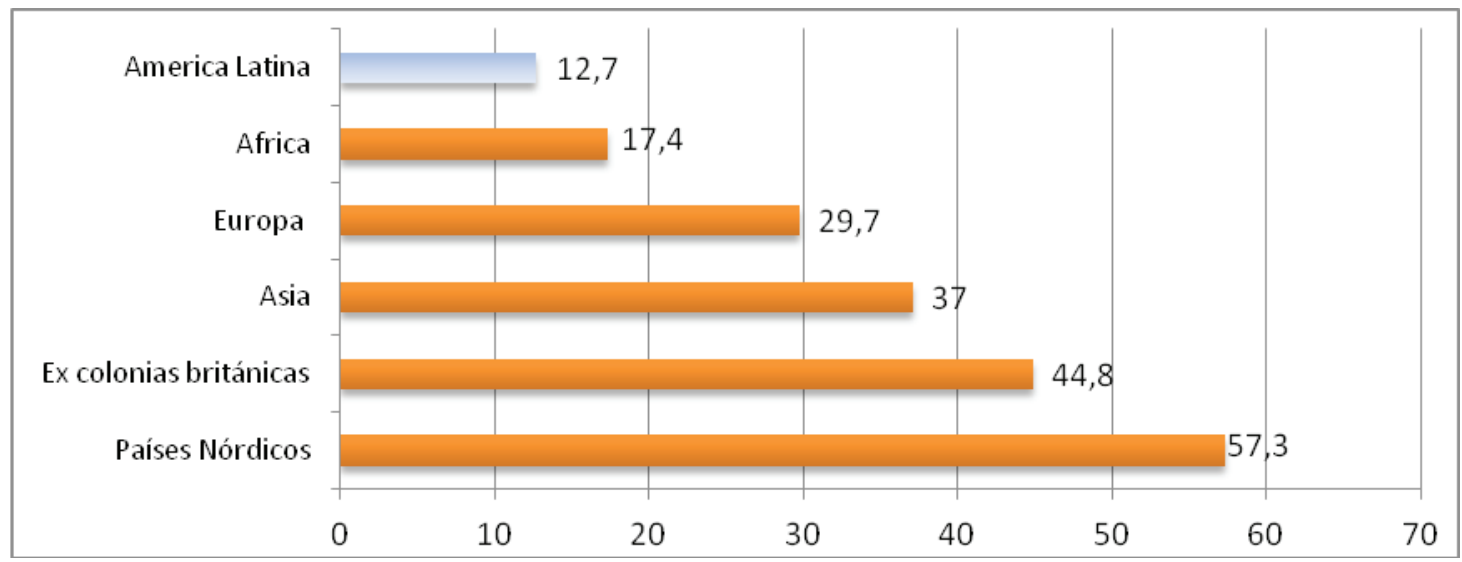

Fuente: World Value Survey

Nota: Países Nórdicos: Países bajos, Suecia y Finlandia; Ex- Colonias británicas: USA, Canadá, Australia and Nueva Zelandia; Europa: Francia, Reino Unido, Italia, España, Polonia, Alemania y Suiza; Asia: Japón, Corea del Sur, China, Taiwán y Tailandia; Africa: Egipto, Marrueco, Etiopía, Ghana, Mali y Ruanda; América latina: Argentina, Brasil, Chile, Colombia, México y Perú.

\section{GRÁFICO 2}

CONFIANZA INSTITUCIONAL POR REGIONES, NIVELES PROMEDIOS. AÑOS 2007-2008.

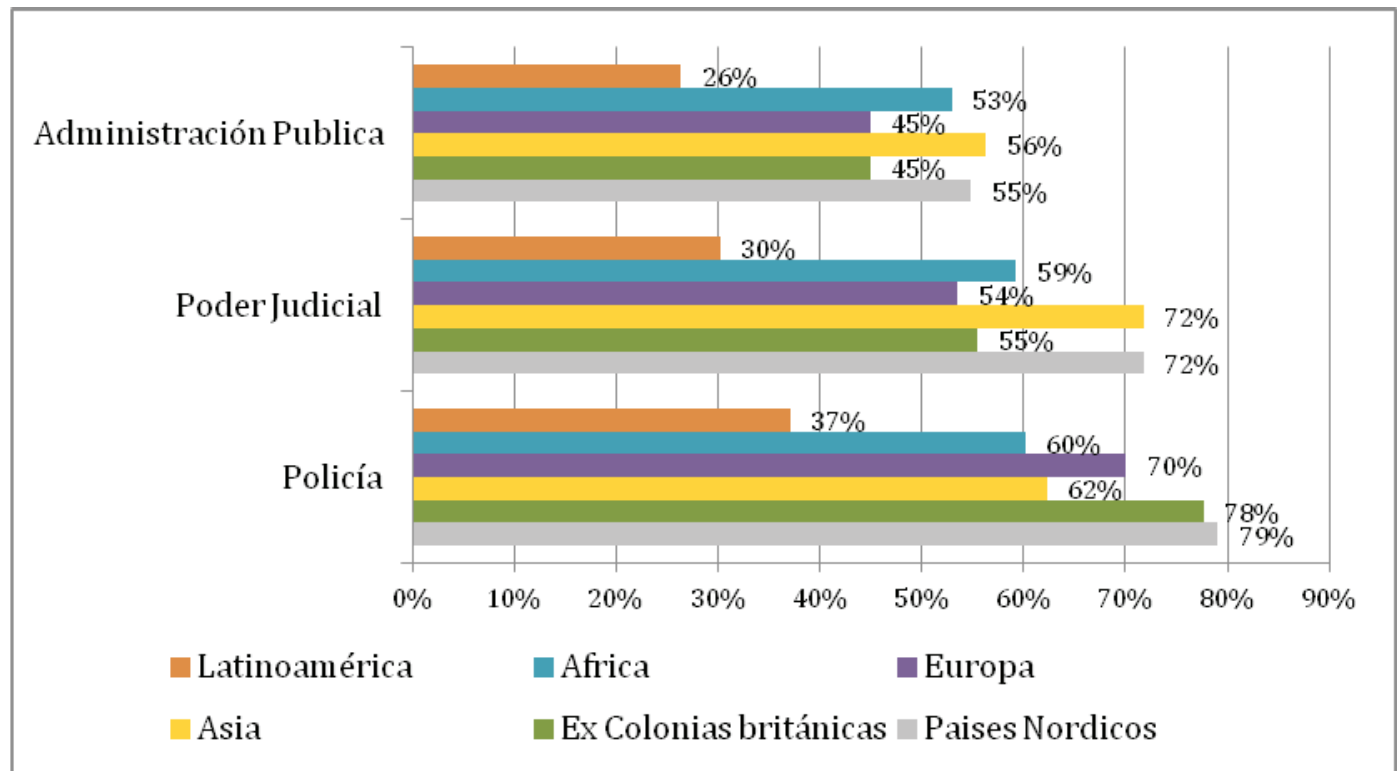

Fuente: World Value Survey (ídem anterior) 
GRÁFICOS 3 Y 4

CONFIANZA SOCIAL Y CONFIANZA EN LA ADMINISTRACIÓN PÚBLICA EN AL. AÑO 2011
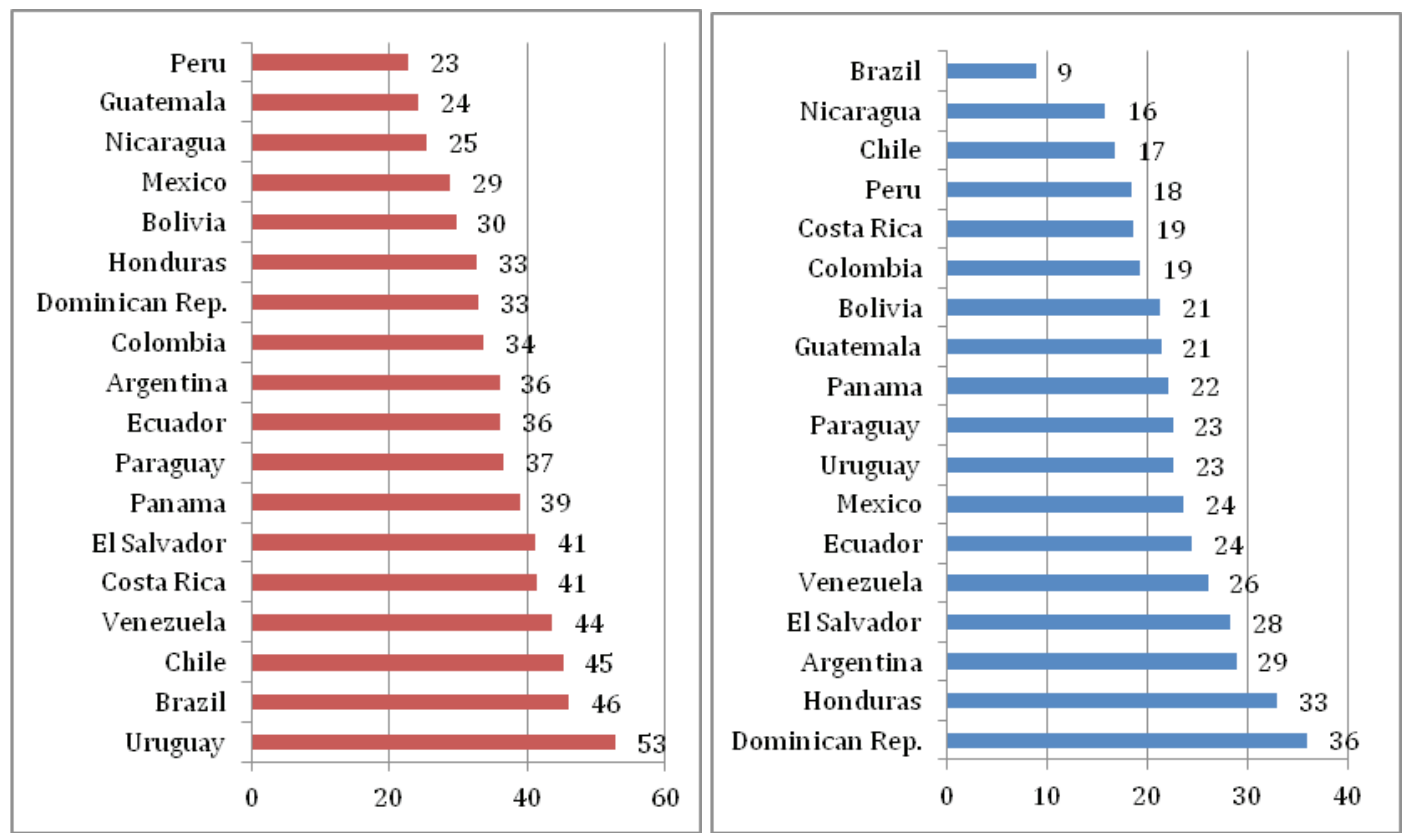

Fuente: Latinobarómetro, 2011

En torno a las instituciones informales, trabajos como el de Escalante en México (2005) dan cuenta, a partir del relato y la narración interpretativa de ciertas características de la región latinoamericana en su conjunto, de cómo la vida política se organiza por medio de relaciones y prácticas regulares con todas las características de un orden efectivo aunque informal que se rige por lógicas de lealtad, reciprocidad, personalismo y señoriales que, en abierta contradicción al Estado de derecho democrático y liberal, son clave para mantener el control político del territorio. También el trabajo de Auyero (2002) describe desde una perspectiva etnográfica las razones en las que se funda el arraigo de una cultura relacional informal denominada clientelar y de cómo tales redes personalizadas de ayuda mutua sirven para satisfacer necesidades en un contexto de baja efectividad estatal y de lejanía con la ciudadanía. Los gráficos que siguen reflejan a la región como la más tolerante a la evasión fiscal en términos comparados, a la par que ilustran un alto porcentaje de tolerancia al favoritismo, especialmente el familiar.

GRÁFICO 5

NIVELES DE TOLERANCIA A LA EVASIÓN FISCAL. AÑOS 2006-2008

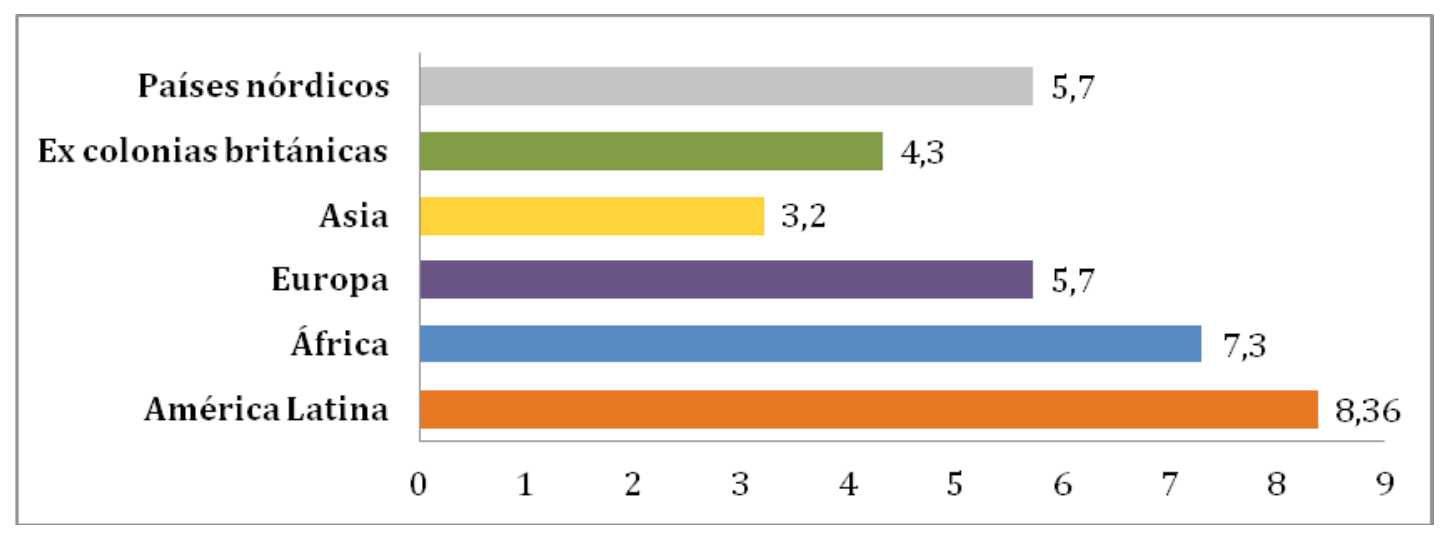

Fuente: World Value Survey

Nota: Se usa la pregunta: Usted cree que siempre se justifica (valor 10) o nunca se justifica (valor 1) evadir impuestos si usted tiene la oportunidad. Se grafica la suma de respuestas de los valores 7 a 10. 
GRÁFICO 6

PORCENTAJES DE LATINOAMERICANOS QUE PIENSAN QUE ES INCORRECTO PERO COMPRENSIBLE QUE EL GOBIERNO OTORGUE TRABAJO A LOS FAMILIARES, A QUIENES LO APOYAN O A MIEMBROS DE SU PARTIDO POLÍTICO. AÑO 2006

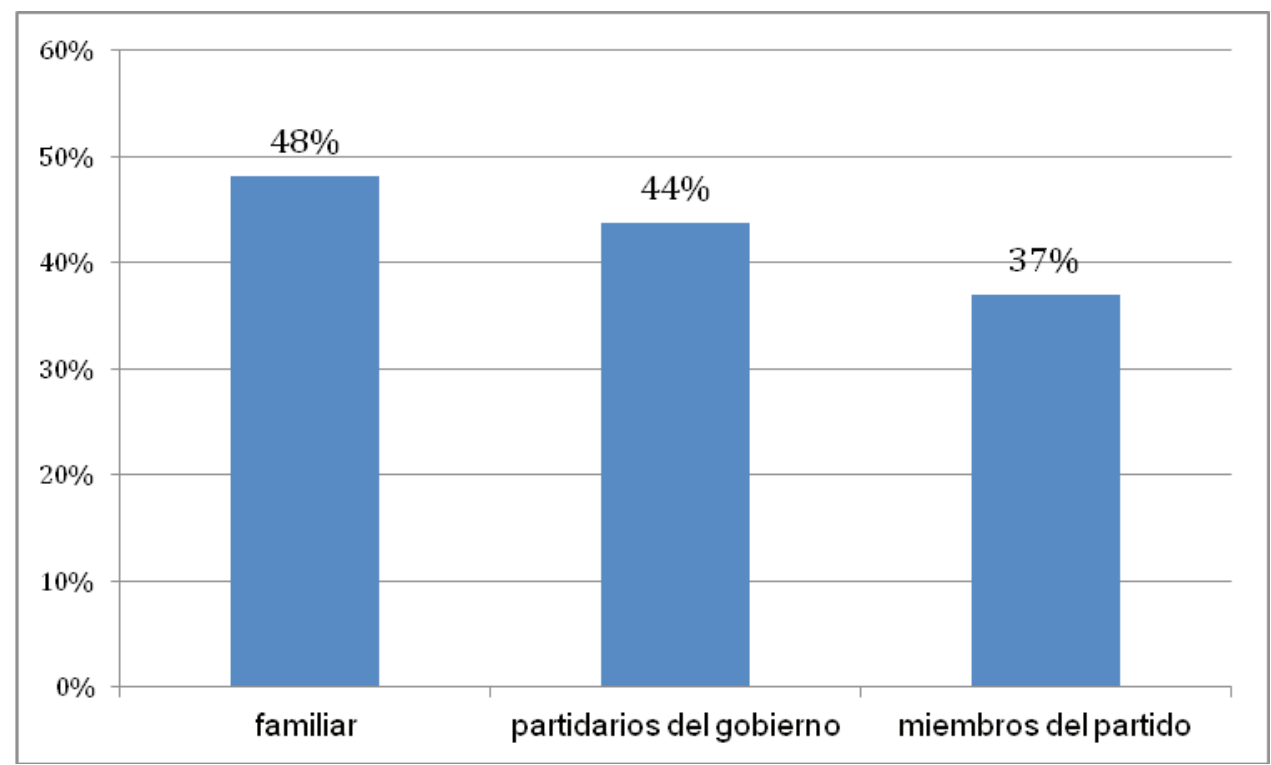

Fuente: Latinobarómetro, 2006 (único año disponible)

Claramente, y más allá de las observaciones que pudieran hacerse en términos comparados con otras regiones, al interior de América Latina se encuentran amplias divergencias. Esto último refleja la necesidad de estudios en profundidad de los países de la región y de las características de los mismos. A tales asuntos se dedican los próximos párrafos.

\subsection{Sobre los estudios de caso: Argentina y Colombia}

El caso argentino busca observar como las políticas públicas impactan en la confianza social. El objetivo es evidenciar modos indirectos y silenciosos en los que las intervenciones públicas van moldeando los escenarios de reproducción de la confianza social y, con ello, condicionan su creación. País históricamente exhibido como exitoso en términos de desarrollo y bienestar social que, presentado como el mejor alumno en la implementación de las políticas de ajuste estructural de orientación mercantilista es quien en pocos años sufre un declive notorio en términos de bienestar y desarrollo. Dichas razones lo convierten en un infeliz laboratorio donde explorar el impacto del trasplante de ciertas políticas públicas en la sociedad y en la confianza social y de las de orientación mercantilistas en sí mismas (Lovuolo \& Barbeito 1998; Sader, 2009). La tabla que sigue ofrece datos agregados de inicios y fin de dicha década. Ello nos permite apreciar el efecto inmediato del paquete de reformas y el paradigma neoliberal por estas auspiciado en la sociedad y la economía.

TABLA N ${ }^{\circ}$

LOS EFECTOS DE POLÍTICAS DE ORIENTACIÓN MERCANTILISTA EN NÚMEROS

\begin{tabular}{|llll|}
\hline & \multicolumn{2}{l}{ Argentina } \\
\cline { 2 - 4 } Indicadores económicos y sociales & 1990 & 2002 & $\begin{array}{l}\text { Variación } \\
\%\end{array}$ \\
\hline PIB - per capita a precios constantes Laspeyres (Penn World Table) & 9432 & 11.239 & 19 \\
\hline Desempleo (SEDLAC) & $6,8(*)$ & 17,9 & 163 \\
\hline Empleo Informal -\% de asalariados sin derecho a pensión- (SEDLAC) & 32,5 & 44.1 & 36 \\
\hline $\begin{array}{l}\text { Salario - Ingreso laboral mensual en pesos constantes de } 2000-(\text { SE- } \\
\text { DLAC) }\end{array}$ & $\begin{array}{l}67,6 \\
(*)\end{array}$ & $\begin{array}{l}598,2 \\
(* * *)\end{array}$ & -12 \\
\hline $\begin{array}{l}\text { Desigualdad -Coeficiente de Gini: distribución del ingreso per capita } \\
\text { por hogares-(SEDLAC) }\end{array}$ & $\begin{array}{l}0,46 \\
(* *)\end{array}$ & 0,53 & 15 \\
\hline
\end{tabular}




\begin{tabular}{|c|c|c|c|}
\hline Polarización social - Índice de bipolarización EGR de Wolfson)(SEDLAC) & $\begin{array}{l}0,40 \\
(* *)\end{array}$ & 0,52 & 30 \\
\hline Pobreza Moderada (SEDLAC) & 33,1 & 57,5 & 74 \\
\hline Pobreza Extrema (SEDLAC) & 6,6 & 27,5 & 317 \\
\hline Confianza social (WVS) & $\begin{array}{l}23,3 \\
(* *)\end{array}$ & $\begin{array}{l}15,4 \\
(* * * *)\end{array}$ & -34 \\
\hline
\end{tabular}

(*) Datos para $1992(* *)$ Datos para $1991(* * *)$ Datos para $2001(* * * *)$ Datos para 1999

Nota: los datos de pobreza moderada y extrema y desigualdad de 1990/1991 corresponde solo a Gran Buenos Aires. Fuente: Elaboración propia en base a: SEDLAC, Socio-Economic Database for Latin America and the Caribbean http://sedlac.econo.unlp.edu.ar/esp/ estadisticas.php (6.05.2012) Penn World Table, http://pwt.econ.upenn.edu/php_site/pwt_index.php (8.03.2012)

World Values Survey, http://www.worldvaluessurvey.org/ (6.05.2012)

Por su parte, el caso colombiano se detiene a describir cómo opera el camino inverso y cómo las instituciones informales impactan en las políticas públicas. Después de la Constitución de 1991, Colombia fue escenario de un profundo cambio institucional que buscó transformar un Estado centralista, presidencialista y bipartidista en una República democrática, participativa y pluralista (Art. 1 de la Constitución Política de 1991). Uno de dichos cambios sustanciales fue el proceso de descentralización política, económica y administrativa, donde se optó por una doble característica institucional de autonomía territorial en un Estado unitario. Esto significó una estructura institucional homogénea para todos los territorios, a pesar de su heterogeneidad física, cultural, social y humana. Este punto se muestra fundamental, pues permite observar cómo cuestiones sociopolíticas de contexto pueden influenciar en el éxito o fracaso de las reformas descentralizadoras en los diversos territorios del país.

Partiendo de dicha homogeneidad formal frente a la heterogeneidad informal, se realiza un estudio cualitativo y comparado del desempeño municipal en dos municipios que aun cuando presentan la misma base institucional alcanzan niveles de desempeño diferenciado. Con este estudio se busca observar qué impacto tiene la institucionalidad informal sobre las posibilidades de éxito o fracaso de una política pública de transferencia de competencias, poniendo especial énfasis en la creación y consolidación de capacidades administrativas para la prestación de servicios en las municipalidades.

Teniendo en cuenta la ya mencionada heterogeneidad de la región, se entiende que lo analizado en estos dos países arroja pistas útiles para entender algunos rasgos generales, a la vez que puede servir como punto de partida para problematizar lo acontecido en otros países latinoamericanos.

\subsection{Argentina: Los efectos de las políticas de orientación mercantilista en la confianza}

Las políticas de orientación mercantilista, tendentes a facilitar y reestructurar las relaciones sociales de modo de ajustarlas a las demandas del capitalismo global fueron etiquetadas y se dieron a conocer en la región como neoliberales. Las ideas y estrategias de acción contenidas en tales políticas transformaron la sociedad argentina no sólo en términos institucionales sino en términos estructurales y culturales (Lo Vuolo \& Barbeito, 1998; Borón, 2002; Alayón \& Grassi, 2002; Portes \& Hoffman, 2003; Basualdo \& Arceo, 2006).²

Nuestra hipótesis es que tales políticas afectaron negativamente la confianza social en tanto reconfiguraron: a) las oportunidades de vida de los grupos sociales y sistemas de estratificación social, b) los valores y creencias a partir de los cuales los sujetos derivan inferencias cognitivas sobre la sociedad y los "otros", c) los espacios públicos que sirven de escenario a experiencias informales de socialización.

2 Bajo un conjunto de políticas de ajustes y reformas, en menos de tres años se privatizaron la mayoría de las empresas públicas, se profundizó la apertura económica con rebaja de aranceles y remoción de barreras no arancelarias, la oferta monetaria se ató a las reservas del Banco Central (acentuándose la tendencia hacia la valorización de las transacciones económicas en la divisa de EEUU), se reprogramó estrictamente la deuda externa, la deuda interna se transfirió hacia futuro mediante colocación de títulos públicos, aumentó abruptamente el endeudamiento internacional, se modificó el régimen laboral reduciéndose la estabilidad y la mayoría de las políticas sociales se mercantilizaron y adquirieron un tinte liberal (Lo Vuolo \& Barbeito, 1998). 
FIGURA 1

POLÍTICAS PÚBLICAS Y CONFIANZA SOCIAL

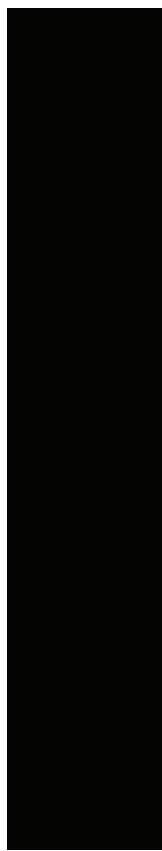

Public Policies and Trust
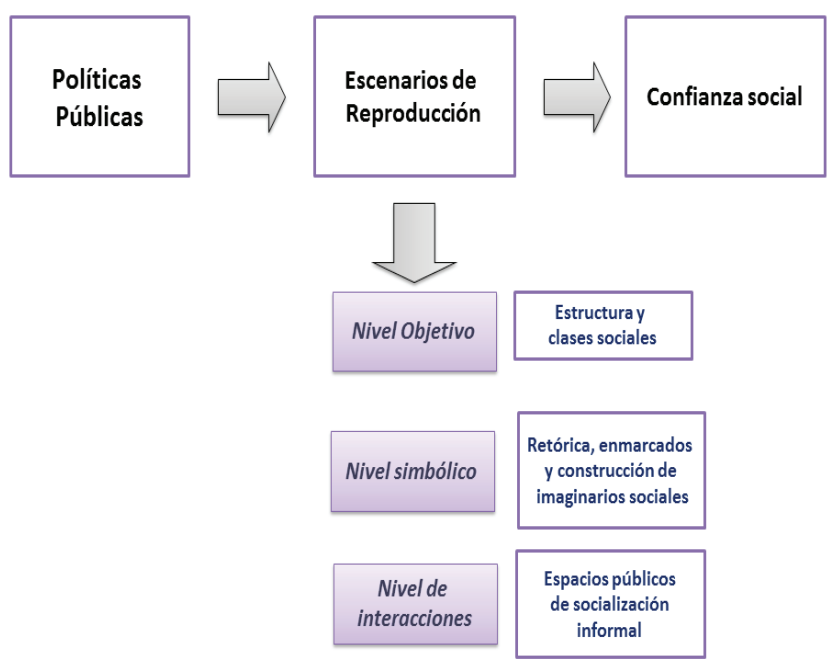

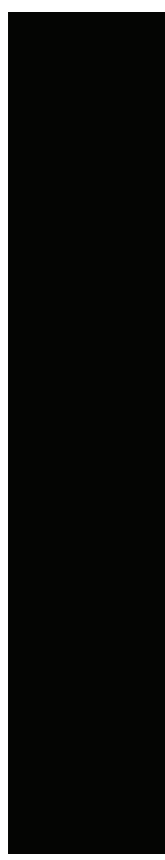

Fuente: Elaboración propia

El primero de los escenarios es la estructura social, esto es: condiciones socio-económicas y peculiaridades de las clases sociales. La hipótesis al respecto sería que, en sociedades donde los canales de ascenso social están abiertos, las divisiones entre las clases son porosas y las clases medias son más abundantes, la confianza social tendría más sentido (Josten, 2005). Por el contrario, en sociedades duales, polarizadas, con fracturas muy marcadas y donde se percibe claramente las divisiones, la confianza tendría menos posibilidades. Asimismo, en tanto que el modo en que el Estado regula el mercado laboral y redistribuye el bienestar involucra una intervención en la distribución del ingreso y afecta en la formación de las clases sociales, es de esperar que reformas laborales que protegen a los trabajadores y garantizan estabilidad en los contratos de trabajo contribuyen a la formación de confianza (Sverke et. Al. 2002; De Witte, 2005; Rothstein, 2008; Anderson \& Pontusson, 2007). Lo acontecido en Argentina, refleja que sucede cuando las políticas van en dirección contraria. Cuando la apuesta estatal es por la flexibilización y desregulación del mercado laboral, la estructura social y redistribución del ingreso se ven afectados negativamente. A la par que aumentó el desempleo, el empleo informal y el temor al desempleo, se incrementó la pobreza de aquellos trabajadores calificados cómo no competitivo, la clase media se rompió y la desigualdad del ingreso se elevó a valores desconocidos (Svampa, 2000; Minujín \& Kessler, 1996). La ruptura de sentidos sociales compartidos en el caso de la fracturada clase media, el aumento de la percepción de exclusión social (en tanto el bienestar está atado a los puestos de trabajo formales) y el temor de quienes aun no habiendo caído y perdido su trabajo, sufren de pensar en dicha posibilidad no puede sino resultar en un coctel negativo para la confianza generalizada en un contexto donde prima la competencia.

El segundo de los escenarios afectado por las políticas de orientación mercantilista alude a las interpretaciones cognitivas y normativas de la realidad social que predominan en un momento dado. Las memorias, narrativas y valoraciones morales que se van construyendo acerca de los "otros" y del "nosotros", son sumamente importantes para el desarrollo de la confianza. Confiar conlleva, por un lado, visualizar a los otros como seres respetables, dignos y honrados, por el otro una idea de "nosotros" que articula el relato y suerte individual con la social. Teniendo en cuenta las funciones expresivas y el poder "performativo" de las intervenciones gubernamentales y el discurso político, es de esperar que el lenguaje, metáforas e instrumentos que se utiliza en una política pública evoque ciertas imágenes y creencias pasadas, actuales y futuras que contribuyan a definir los mundos sociales que las personas experimentan y sus estructuras mentales (Frykman, et. al 2009; Rothstein \& Uslaner, 2005; Jobert, 1989; Fischer, 2003). Así, investigaciones sobre el tema sugieren que, el uso de ciertas ideas y herramientas asociadas a la colectivización, solidaridad y universalismo son favorables a la formación de la confianza en tanto genera conciencia sobre la interdependencia social y ayudan a visualizarnos como partes de un todo (De Swaan, 
1992; Rothstein, 2000 y 2008). Por el contrario, si lo que prima es el aseguramiento individual del bienestar y una satisfacción más dependiente del mercado, las ideas de merecimiento y responsabilidad propia son las que se instalan, dificultando la percepción colectiva (Tamilina, 2009; Patulny, 2009; Güell, 2002). Las políticas neoliberales en Argentina fueron en este último sentido instalando discursiva y fácticamente modelos privatistas y mercantilistas de gestión. Al paso que se desmontaban responsabilidades compartidas y se liberalizaba el modelo de bienestar social existente, se fue arraigando en el inconsciente colectivo ideas de merecimiento social. Este giro mercantilista en los discursos y políticas públicas está bien sistematizado en el trabajos de Bercholc, (2011) quien tras una investigación de los discursos presidenciales de apertura de la Asamblea Legislativa en la Argentina ${ }^{3}$ en los 90', demuestra la aparición y recurrencia en el discurso de nuevas palabras como: competitividad, ajuste, sector privado, especulación, privatización, economía popular de mercado y, por el otro, la desaparición de otras palabras como industria y obras públicas. El Estado pasa a ser identificado como el gran culpable y el mercado como el mesías, lo cual en términos prácticos y de bienestar significa una transferencia de responsabilidad. Específicamente, las políticas de reforma de la sanidad (programa de autogestión hospitalaria) educación (ley federal de educación) y pensiones (privatización del sistema), además de apelar a estas ideas y terminología, introducen herramientas y estrategias en pos de privatizar, mercantilizar e individualizar el bienestar (Centralogo \& Devoto, 1998; Acuña \& Chudnovsky, 2002; Márquez, 1996; Pelayes, 2000; Nino, 2003; Centrálogo \& Grushka, 2004). La privatización del bienestar supone así una naturalización y legitimación de las diferencias sociales que impuso una sujeción de corte moral bajo la cual el individuo interiorizó su éxito o fracaso. Corolario de ello, el sentido de pertenencia social, la idea de "nosotros" y la visualización de un destino común resintieron la cohesión social repercutiendo negativamente en la formación de la confianza social.

El tercer y último escenario intenta demostrar que la confianza social además de depender de las creencias e ideas también lo hace de las interacciones cotidianas, esto es: experiencias de solidaridad y empatía desarrolladas en el día a día. Resultado de ello, los espacios públicos se convierten en contextos relevantes para el análisis en tanto es allí donde los individuos aprenden a vivir en sociedad y a sentirse parte de la comunidad. En términos prácticos, la socialización entre diferentes en condiciones de igualdad puede contribuir a que los sujetos disipen prejuicios, desarrollen empatía, pierdan el miedo al diferente y fortalecer los sentimientos y obligaciones morales y solidaridad respecto a grupos menos privilegiados. En suma, tales contactos son útiles para expandir la información acerca del mundo que nos rodea y las preferencias de los otros, cuestiones estas que reducen la incertidumbre y el riesgo de confiar (Bourdieu, 2001; Portes \& Landolt, 2000; Kaztman, 2003; Rothstein \& Uslaner, 2005; Rothstein, 2008).

Dicho esto, y tomando como referencia los cambios urbanos que experimenta la ciudad y la composición social de la escuela, se sostiene que: si el acceso a tales espacios públicos es generalizado y promueven el encuentro entre desemejantes habrá más oportunidades de desarrollar actitudes pro-sociales y más oportunidades para que surja la confianza. Por el contrario, si tales escenarios se deterioran, se segmentan o fragmentan, las oportunidades de interacción entre grupos y categorías sociales disminuyen y, con ello, se profundizan las fronteras sociales y la confianza pierde oportunidades (Sennet, 1975, 1978; Wacquant, 2007; Borja, 2005; Gradstein \& Justman 2000 ).

En Argentina, lo que se observa es un desvanecimiento de los espacios públicos y un incremento de la segregación espacial y social. La ruptura de la clase media y la reconfiguración de los imaginarios sociales en términos privatistas y mercantilistas se vieron reforzada por un mercado inmobiliario que buscaba ofrecer servicios especiales a los ganadores y que reforzó la expulsión material y simbólica de los perdedores. La autoexclusión en barrios cerrados en paralelo al incremento de la población en asentamientos irregulares no pudo sino que aumentar la segregación espacial y social. En paralelo, la segmentación de la escuela pública y la huida de la clase media hacia la enseñanza de gestión privada empeoraron la fragmentación social, desdibujando la función integradora e igualadora de la escuela. Políticas sectoriales desreguladoras radicalizaron estos fenómenos.

Es evidente que en la relación pendular entre confianza y políticas públicas mucho se ha escrito sobre como la confianza condiciona el éxito de las sociedades y la eficacia de Estado, pero poco se ha analizado en relación a cómo el Estado y las políticas públicas influían en la creación o destrucción de la confianza, especialmente en América Latina. Atento a ello, nos propusimos desentrañar caminos en virtud de los cuales las políticas públicas influyeran en la confianza social de modos indirectos aunque no por ello menos importantes y duraderos. A fin de visualizar esto, elegimos un corpus de políticas que se implementaron en una franja de tiempo mensurable y que suelen ser promovidas como modernizadoras. En un par de párrafos presentamos los principales hallazgos que en

3 Discursos prescriptos por la Constitución Nacional, son las verbalizaciones más importantes, en términos jurídicos e institucionales y es donde la Presidencia inaugura el período anual de sesiones parlamentarias a la par que se exterioriza la visión que de la realidad tiene y/o pretende construir. 
la investigación más amplia hallamos sobre el impacto negativo de las políticas de inspiración neoliberal tuvieron en la formación de la confianza en Argentina.

En tanto las políticas públicas se enfocan en el libre juego de las fuerzas de mercado, las estructuras sociales se tornaron menos igualitarias, las clases medias se redujeron, los imaginarios se privatizaron y los espacios públicos se achicaron. A raíz de ello, los encuentros casuales con el diferente se volvieron más improbables, las sociabilidades familiares se tornaron homogéneas e íntimas y la confianza social perdió terreno aumentando la confianza particularizada y el asociacionismo de resistencia. Los lazos familiares que emergieron ayudaron a manejar la ansiedad y los miedos reduciendo el impacto negativo de las transformaciones acaecidas pero no contribuyeron necesariamente al desarrollo de sentidos y responsabilidades colectivas y por ello resultaron negativas en lo que refiere a la formación de confianza, creando ambientes no "trust friendly".

\subsection{Colombia: gobiernos locales e instituciones informales}

En este apartado se busca entender qué aspectos de la institucionalidad informal pueden afectar el desempeño diferenciado de una misma política pública. Para tal fin, nos enfocamos en dos municipios colombianos que con características similares a nivel institucional formal, poblacionales similares, competencias en sanidad y educación y una evolución incremental de sus ingresos totales, pero que presentan resultados divergentes en materia de desempeño político (Tabla 2).

TABLA 2

COMPARATIVO DATOS MUNICIPIOS SELECCIONADOS

\begin{tabular}{|lcc|}
\hline & Facatativá (Cundinamarca) & $\begin{array}{c}\text { Ciénaga } \\
\text { (Magdalena) }\end{array}$ \\
\hline Categoría & Tercera (2009) & Cuarta (2009) \\
\hline Población & 119.849 (2010) & 103.066 (2010) \\
\hline Certificaciones & Sanidad y Educación & Sanidad y Educación \\
\hline Ingresos Totales (2000) & 16.910 millones COP & 12.719 Millones COP \\
\hline Ingresos Totales (2010) & 71.974 millones COP & 101.805 Millones COP \\
\hline \% Población NBI (1993) & 21,66 & 52,72 \\
\hline \% Población NBI (2010) & 13,09 & 43,81 \\
\hline ITM* 2008-2009 & 75,1 (Moderado) & 39,7 (Muy alto) \\
\hline Desempeño político & Premios Mejor Plan de Desarro- & Informe MOE: Riesgo EXTREMO \\
\hline
\end{tabular}

Fuente: Elaboración propia partiendo de datos DANE, DNP, TPC, MOE y CL. *Índice de Transparencia Municipal, creado por Transparencia por Colombia que mide el nivel de riesgo municipal ante actos de corrupción.

Como se observa en la tabla, mientras Facatativá ha sido referencia nacional, y premiado tanto a Mejor Alcalde de ciudad intermedia como a Mejor Plan de Desarrollo 2008-2011 (premios Colombia Líder), en Ciénaga saltaron las alarmas de la Misión de Observación Electoral en su estudio de 2011 sobre riesgos ante el fraude y la violencia. (Misión de Obervación Electoral, 2011; Transparencia por Colombia, 2010). Pero la diferencia más visible se centra en sus niveles de desempeño municipal: mientras Facatativá ha estado de manera constante en lo alto de las mediciones a nivel nacional, Ciénaga ha tenido un promedio muy inferior en las últimas mediciones realizadas por el Departamento Nacional de Planeación (Tabla 3).

TABLA 3

ÍNDICE DE DESEMPEÑO MUNICIPAL 2007-2010 CASOS SELECCIONADOS

\begin{tabular}{|c|c|c|c|c|c|}
\hline Municipio & IDM 2007 & IDM 2008 & IDM 2009 & IDM 2010 & Promedio \\
\hline Facatativá & 82,08 & 90,32 & 84,37 & 84,4 & 85,29 \\
\hline Ciénaga & 33,49 & 33,07 & 57,41 & 78,5 & 50,62 \\
\hline
\end{tabular}

Fuente: Departamento Nacional de Planeación, Informes IDM de 2007 a 2010

¿Cuál es la razón de tan marcada diferencia? Durante la investigación de campo, la cual contó con una serie de 37 entrevistas semi-estructuradas a funcionarios públicos y miembros de organizaciones sociales en ambos municipios, se evidenciaron varias explicaciones que pueden resumirse en la existencia de sistemas de incentivos com- 
pletamente diferenciados. Específicamente, si los políticos locales ven a los procedimientos formales como mejor camino para lograr sus objetivos, harán que las políticas públicas sean acordes a ellos, pero si los procedimientos son vagos e irrelevantes, o si existe la posibilidad de no ser sancionados por su incumplimiento, las normas informales prevalecerán y las políticas públicas tendrán nula capacidad de revertir esta situación.

En el caso de Facatativá, dada su cercanía física con la capital de la República (40 km), existen mayores incentivos para consolidar las formas y los protocolos exigidos por los niveles superiores de gobierno, puesto que dicho cumplimiento de la forma se materializa en el aprovechamiento de sus posibilidades de negociación con el nivel departamental, y sobre todo con el nivel nacional. En efecto, los municipios aledaños a Bogotá tienen un nivel muy alto en sus índices de desempeño: Facatativá, Fusagasugá, Zipaquirá, Chía, Mosquera, Madrid, Funza, y Cajicá, todos mantienen promedios de IDM de alrededor de 80 sobre 100 puntos. ${ }^{4}$ Dicha ventaja comparativa de proximidad física del municipio al centro político y administrativo del país se genera en dos vías: en primer lugar, la posibilidad de negociación de recursos es más directa, lo que genera a su vez una necesidad de seguir la formalidad, de generar procesos administrativos claros que vayan de acuerdo con las directrices nacionales, en aras de aprovechar dicha posibilidad. Con el tiempo, el énfasis que se le otorga a la formalidad y al protocolo ha generado ciertas capacidades administrativas, sumado al reconocimiento del municipio entre distintos organismos departamentales y nacionales, que permiten enfrentarse a convocatorias y a proyectos con destinación de recursos de manera más eficiente que otros municipios con sus mismas características. En otras palabras, la cercanía física con los entes nacionales, y la posibilidad de negociación que ésta conlleva, genera una estructura de incentivos que empuja al cumplimiento de las leyes como la vía más conveniente de acción, puesto que aun cuando sean cambios únicamente protocolarios, éstos implican a su vez un mejoramiento de las capacidades burocráticas del municipio. El juego se presenta como ganador para ambos actores, locales y nacionales.

En Ciénaga, Magdalena, la cuestión es muy distinta, pues la lejanía de la capital es más que evidente y no solo en términos geográficos. En un municipio donde los recursos se han incrementado exponencialmente, los actores tienen la sensación que el vínculo del gobierno con ellos es frio y puramente interesado 5 . Sin embargo, no existen mecanismos que controlen el uso de las enormes regalías y, por el contrario, los actores creen que la corrupción es la regla. La percepción generalizada es que las instituciones informales clientelares son las que dominan la realidad del municipio ${ }^{6}$. Como resultado de todo ello, y pese al incremento de recursos fiscales, en 2008 la municipalidad de Ciénaga tuvo que declararse en quiebra. Nadie fue arrestado o perseguido procesalmente ni respondió políticamente por el desastre financiero. Los lazos clientelares salieron fortalecidos y legitimados como reglas que rigen, destruyendo algún atisbo de confianza en las instituciones.?

Tal vez más preocupante que la prevalencia de una élite política que utiliza los puestos del gobierno municipal de manera patrimonialista, son los efectos perversos que esto conlleva: un entramado de normas aceptadas de manera informal que van en contravía con la esencia de las instituciones legales, entrando en competencia directa con ellas. En este sentido, los entrevistados que trabajaban en la alcaldía mencionaban mecanismos de corrupción de conocimiento general y que se llevan a cabo de manera regular: para un permiso se necesita tanto, para un contrato, tanto; para evitar controles, tanto; etc. Es tal el nivel de "normalidad" de dicha situación que aún con la llegada de nuevos liderazgos, por fuera de la élite política tradicional, estos sistemas de dádivas y favores informales prevalecen, puesto que se entiende que es así como funcionan las cosas. La ciudadanía no entiende los mecanismos de control como tales, sino como mecanismos de negociación con la alcaldía, en aras de obtener mayores beneficios personales o para su grupo de interés.

En conclusión, en el caso de los gobiernos locales estudiados, la relación entre formalidad e informalidad ha generado un sistema de incentivos de acción totalmente diferenciado entre los líderes políticos de estos dos municipios: mientras que en el caso de Facatativá el seguimiento de la formalidad se muestra como el mejor camino para obtener beneficios para las autoridades locales (reconocimiento político no solo local sino regional y nacional), lo cual ha desembocado tanto en beneficios para la administración (consolidación de procesos) como mejoramiento en la calidad de vida de la ciudadanía (mejores servicios públicos y posibilidades de participación), en el caso de Ciénaga los líderes optan casi de manera generalizada por vías informales, basadas en visiones tradi-

4 Aun cuando Colombia es un país descentralizado, la mayoría de los gobiernos locales dependen de fondos y transferencias del gobierno nacional (Jiménez, 2001) (Faguet \& Sánchez, 2009).

5 Ciénaga tiene tres diferentes y privatizados puertos marítimos donde las compañías internacionales de carbón aprovisionan grandes cargueros, hecho esto que generan enormes regalías al municipio.

6 "En el caso de Ciénaga y buena parte del Departamento [del Magdalena] lo que opera es una anomia social en donde cada quien establece como referencia sus propios criterios (...) Y en buena medida eso está explicado es por la ausencia de la mano del Estado" (Entrevista 22, 29 de marzo de 2012).

7 Un 80\% de la población en Ciénaga desconfía de las instituciones formales y los políticos (Universidad del Magdalena, 2010) 
cionales de autoridad y cacicazgo que se muestran muy beneficiosas a manera individual, aunque al final resulten absolutamente desastrosas para el colectivo ${ }^{8}$. A pesar de los esfuerzos de algunas organizaciones, las posibilidades de presión ciudadana en Ciénaga son muy reducidas, impidiendo esto la existencia de un contrapeso social, algo que, sumado a lo anterior, hace que los incentivos para mantener las prácticas clientelares sean tan fuertes que a la larga resulta irracional seguir las normas formales establecidas desde el nivel central.

Esta diversidad de los sistemas de incentivos entre los casos estudiados se explica al revisar el diseño institucional rígido y homogéneo de la descentralización colombiana, el cual no tuvo en cuenta los diversos y complejos contextos en los que se debe implementar la política pública (Departamento Nacional de Planeación DNP, 2007; ICP-UNAL, 2012). Esto se hace especialmente relevante al no tener en cuenta las reglas no formales preexistentes en los territorios, las cuales marcan un sistema alternativo de comportamiento que puede apoyar (como en el caso de Facatativá) o entrar en competencia (como en el caso de Ciénaga) con las metas últimas de la reforma institucional.

\section{REFLEXIONES E INTERROGANTES}

Más allá del interés académico que pueden suscitar estas cuestiones, las mismas resultan claves en términos de políticas públicas en tanto condicionan el logro de los objetivos y metas que las sociedades se proponen alcanzar. Sin embargo, éste ha sido un tema que no ha sido del todo desarrollado dentro de la academia, a pesar de las serias implicaciones de la informalidad en un contexto como el latinoamericano, región que política e ideológicamente ha experimentado grandes cambios formales pero que aún hoy mantiene algunas prácticas sociales alejadas de los valores democráticos.

Claramente, lo acaecido en Argentina responde a múltiples causas y puede ser leído de diferentes modos, pero creemos que esta es una manera válida de verlo y que apunta a profundizar en el análisis de los "outcomes" de las políticas públicas o impactos de segundo orden de la misma en un variable tan difícil de estudiar pero a la vez reconocida como fundamental y necesaria para el desarrollo exitoso de las sociedades. De modo que, el interés de este análisis no es extrapolar reflexiones para el resto de la región sino señalar nuevos lugares que sirvan de punto de partida para futuros análisis sobre el tema de la confianza, sobre como las políticas públicas afectan la misma y sobre las peculiaridades de una región como la latinoamericana, donde la confianza escasea pero el desarrollo, la democracia y la eficiencia de Estado son un desafío que urge y demanda, paradójicamente ciertos niveles de confianza social.

En el caso de Colombia, el estudio comparado de experiencias locales resulta de sumo interés en un escenario donde aún se debaten los efectos de la descentralización y el impacto de reformas legales. Recuperar y poner sobre la mesa el papel que juegan las instituciones informales y las motivaciones de los agentes gubernamentales complejiza el análisis y contribuye a explicar los resultados hallados.

En este sentido, las investigaciones en profundidad en tanto combinan herramientas cuantitativas y cualitativas de investigación son capaces de generar conocimiento científico y se vuelven fundamentales para entender cómo las políticas públicas pueden transformar comportamientos sociales y como éstos influyen en el impacto real de las acciones del Estado. En suma: las instituciones informales y la confianza son conceptos difusos que carecen de indicadores claros pero que pese a ello es necesario incorporar y mantener en la agenda de análisis de las políticas públicas.

\section{BIBLIOGRAFÍA}

Acuña, C., y Chudnovsky, M. (2002). El Sistema de Salud en Argentina. Buenos Aires: Documento 60. Universidad de San AndrésCentro de Estudios para el Desarrollo Institucional. Fundación Gobierno y Sociedad.

Alayón, N., y Grassi, E. (2002). Neo- liberalims in Argentine. Social policy, welfare and the conditions for the development of social work. En I. Fergurson, M. Lavalette, \& E. Whietmore, Globalisation and Social Work: Perspectives from the left. London : Routledge .

Almond, G., y Verba, S. (1970). La Cultura Cívica. Estudios sobre la participación política democrática en cinco naciones. Madrid: Euramericana.

Anderson, C., y Pontusson, J. (2006). Workers, worries and welfare states: Social protection and job insecurity in 15 OECD countries. European Journal of Political Research, 46(2), 211-235.

8 Un ejemplo de estas prácticas personalistas son los llamados "elefantes blancos": megaproyectos de infraestructura que terminan endeudando al municipio, enriqueciendo a unos pocos, y condenando a la población a pagar un bien inservible. Ciénaga cuenta con el suyo: el Polideportivo Municipal, un proyecto que ha vaciado las arcas durante los últimos cinco años y que hoy en día sigue siendo un terreno inutilizable. 
Auyero, J. (2002). Clientelismo Político en Argentina: doble vida y negación colectiva. Perfiles Latinoamericanos, FLACSO(20), 33-52.

Basualdo, E., y Arceo, E. (. (2006). Neoliberalismo y sectores dominantes. Tendencias globales y experiencias nacionales. Buenos Aires: CLACSO.

Bercholc, J. (2011). El contenido económico de los discursos presidenciales en la Argentina -1983-2011. . Buenos Aires: Documento de investigación. Facultad de Derecho. Universidad de Buenos Aires.

Boix, C., y Postner, D. (2000). Capital social y Democracia. Revista Española de Ciencia Política, 1(2), $159-185$.

Borja, J. (2005). La ciudad conquistada. Madrid: Alianza.

Borón, A. (2003). Después del saqueo: el capitalismo latinoamericano a comienzos del nuevo siglo. En A. Borón, Estado, capacidades y democracia en América Latina (págs. 15-38). Buenos Aires: CLACSO.

Bourdieu, P. (2001). El capital social. Apuntes provisionales. Zona Abierta(94/95), 83-87.

Bowles, S., y Gintis, H. (2002). Social Capital and Community Governance. Economic Journal, 102(483), 419-436.

Burky, S., y Perry, G. (1998). Más allá del Consenso de Washington. La hora de la Reforma Institucional, Estudios del Banco Mundial sobre América Latina y el Caribe. Washington DC: Banco Mundial.

Centralogo, O., yDevoto, F. (1998). Reformas en la política de salud en Argentina durante los años noventa, con especial referencia a la equidad. Buenos Aires: Serie Estudios 27, CECE.

Centrálogo, O., y Grushka, C. (2004). Sistema previsional argentino: crisis, reforma y crisis de la reforma. Santiago de Chile: CEPAL.

CEPAL. (2002). Agenda social capital social: sus potencialidades y limitaciones para la puesta en marcha de políticas y programas sociales. CEPAL- Naciones Unidas.

De Swaan, A. (1992). A cargo del Estado. Ediciones. Barcelona: Pomares-Corredor.

De Witte, H. (2005). Job insecurity: review of the international literature on definitions, prevalence, antecedents and consequences. SA Journal of Industrial Psychology, 31(4), 1-6.

Departamento Nacional de Planeación DNP. (2007). Fortalecer la descentralización y adecuar el ordenamiento territorial. Propuesta para discusión. Bogotá: DNP.

Escalante, F. (2005). Ciudadanos imaginarios. México: Colegio México.

Evans, P. (1996). Government Action, Social Capital and Development: Reviewing the Evidence on Synergy. World Development, 24(6), 1119-1132.

Evans, P. (2003). El hibridismo como estrategia administrativa: combinando la capacidad burocrática con las señales de mercado y la democracia deliberativa. Reforma y Democracia. Revista del CLAD, 25, 1-15.

Fischer, F. (2003). Reframing Public Policy. Discursive Politics and Deliberative Practices . Great Britain: Oxford University Press.

Frykman, J. e. (2009). Sense of Community. Trust, Hope and Worries in the Welfare State. Ethnologica Europaea, 39(1), 7-46.

Gradstein, M., y Justman, M. (2000). Human Capital, Social Capital, and Public Schooling. European Economic Review, 44, 879-890.

Granovetter, M. (1973). The Strength of Weak Ties. American Journal of Sociology, 78(6), 1360-1380.

Güell, P. (2002). ¿Quién le apuesta al Capital Social en América Latina? . Conferencia pronunciada en Universidad del Rosario. Bogotá, Colombia.

Güemes, M. C. (2011). Herramientas para un análisis del impacto de las políticas públicas en la confianza social. (I. U. Gasset, Ed.) Circunstancia.(26), (publicación electrónica).

Herreros, F., y Criado, H. (2001). El problema de la formación del capital social. Estado, asociaciones voluntarias y confianza generalizada. Zona Abierta(94/95), 201-231.

ICP-UNAL. (Diciembre de 2012). Observatorio Legislativo - Boletín 214. Recuperado el 2013 de Diciembre de 30, de www.icpcolombia.org/archivos/observatorio/boletin_2014 
Jobert, B. (1989). The Normative Frameworks of Public Policy. Political Study, XXXVII, 376-386.

Josten, S. D. (2005). Middle-Class Consensus, Social Capital and the Mechanics of Economic Development. Working Paper No 36/2005 from Helmut Schmidt University, Hamburg.

Katzman, R. (18 y 19 de Diciembre de 2003). Capital social y sociedad civil en America Latina. Seminario BID sobre La Agenda Ética Pendiente en América Latina. Montevideo, Uruguay.

Kumlin, S., y Rothstein, B. (2003). Investing in Social Capital:The Impact of Welfare State Institutions. Annual Meeting of the American Political Science Association. Philadelphia, USA.

Levi, M. (1998). A State of Trust. En V. Braithwaite, \& M. Levi, Trust and Governance (págs. 77-101). New York: Russel Sage Foundation.

Linares, F. (2007). El problema de la emergencia de normas sociales en la acción colectiva. Una aproximación analítica. Revista Internacional De Sociología (RIS), LXV (46), 131-160.

Lo Vuolo, R., \& Barbeito, A. (1998). La nueva oscuridad de la política social. Del Estado populista al neoconservador. Buenos Aires: Miño y Dávila- CIEPP.

Márquez, Á. (1996). La quiebra del sistema educativo argentino. Montevideo: Libros del Quirquincho.

Meny, I., y Thoenig, J. C. (1992). Las políticas públicas. Barcelona: Ariel.

Minujin, A., \&yKessler, G. (1995). La nueva pobreza en la Argentina. Buenos Aires: Planeta.

Nino, M. (2003). Privatización 2- Reforma Previsional-La subordinación del interés público en el proceso democrático de decisión y negociación en la Argentina. Realidad Económica(195).

North, D. (2006). Instituciones, Cambio Institucional y Desempeño Económico. México: FCE.

Offe, C. (1999). How can we Trust our Fellow Citizens? En M. (. Warren, Democracy and Trust (págs. 42-87). Cambridge: Cambridge University Press.

Ostrom, E., y Ahn, T. (2003). Una perspectiva del capital social desde las ciencias sociales: capital social y acción colectiva. Revista Mexicana de Sociología, 1, 155-233.

Patulny, R. (2005). Social Rights and Social Capital: Welfare and Co-operation in Complex Global Society. American Review of Public Affairs, 6(1), 59-75.

Pelayes, O. (2000). La hipocresía neoliberal: las nuevas formas de privatización de la educación como utopía democratizadora. Revista Herramienta(12).

Portes, A., y Hoffman, K. (2003). Las estructuras de clase en América Latina: composición y cambios durante la época neoliberal. Desarrollo Económico IDES, 43(171), 355-385.

Portes, A., y Landolt, P. (2000). Social Capital: Promise and Pitfalls of Its Role in Development. Journal of Latin American Studies, 32(2), 529-547.

Putnam, R. (1993). Making Democracy Work. Civic traditions in Modern Italy. Princeton: Princeton University Press.

Putnam, R. (2001). La comunidad próspera. El capital social y la vida pública. Zona Abierta, 94-95, 89-104.

Rothstein, B. (2000). Trust, social dilemmas, and the strategic construction of collective memories. Journal of Theoretical Politics, 12, 477-501.

Rothstein, B. (2008). Is the Universal Welfare State a Cause or an Effect of Social Capital? QOG Working Paper Series 16.

Rothstein, B., y Stolle, D. (2002). "How Political Institutions Create and Destroy Social Capital:An Institutional Theory of Generalized Trust . Project on Honesty and Trust: Theory and Experience in the Light of Post-Socialist Experience, . Budapest.

Rothstein, B., \& Uslaner, E. (2005). All for All. Equality, Corruption, and Social Trust. World Politics, 58, 41-72.

Sader, E. (2009). Postneoliberalism in Latin America. Development Dialogue, 51, 171-179.

Sennet, R. (1975). Vida urbana e identidad personal. Barcelona: Ediciones Península.

Sennet, R. (1978). El declive del hombre público. Barcelona: Ediciones Península. 
Stiglitz, J., Sen, A., \&yFitoussi, J. P. (2008). Report by the Commission on the Measurement of Economic Performance and Social Progress". Disponible en: www.stiglitz-sen-fitoussi.fr.

Svampa, M. (2000). Clases Medias, Cuestión Social y Nuevos Marcos de Sociabilidad. Punto de Vista(67).

Sverke, M. e. (2002). No security: A meta-analysis and review of job insecurity and its consequences. Journal of Occupational Health Psychology, 7(3), 242-264.

Tamilina, L. (2009). The Impact of Welfare State Development on Social Trust Formation: an Empirical Investigation. Electronic Journal of Knowledge Management , 7(4), 501-508.

Wacquant, L. (2007). Los condenados de la ciudad. Gueto, periferias y Estado. Buenos Aires: Siglo XXI. 